\title{
Pontos de Corte para a Aptidão Cardiorrespiratória e a Triagem de Fatores de Risco para Doenças Cardiovasculares na Infância
}

\section{Cardiorespiratory Fitness Cut Offs Points and Cardiovascular Risk Factors Screening at Infancy}

Gabriel Gustavo Bergmann' Adroaldo Cezar Araujo Gaya² Ricardo Halpern ${ }^{3}$

Mauren Lúcia de Araújo Bergmann ${ }^{3}$ Ricardo Rodrigo Rech ${ }^{3}$ Cristine Boom Constanzi ${ }^{3}$ Lidiane Requia Alli ${ }^{3}$

1. Curso de Licenciatura em Educação Física da Universidade Federal do Pampa (Unipampa); Projeto Esporte Brasil (Proesp-BR)

2. Programa de Pós-Graduação em Ciências do Movimento Humano da Universidade Federal do Rio Grande do Sul (UFRGS); Projeto Esporte Brasil (Proesp-BR).

3. Programa de Pós-Graduação em Saúde Coletiva da Universidade Luterana do Brasil (ULBRA).

Endereço para correspondência: Rua das Hortênsias, 465, Igara II 92410-580 - Canoas - RS

E-mail: gabrielgbergmann@gmail.com

\section{RESUMO}

O principal objetivo deste estudo foi analisar a validade e propor novos pontos de corte para aptidão cardiorrespiratória de escolares de sete a 12 anos de idade. A amostra foi constituída por 1.413 escolares. A aptidão cardiorrespiratória foi medida através do teste de corrida/caminhada de nove minutos. O colesterol total e as pressões arterial sistólica e diastólica foram medidas e através delas foram criadas referências de fatores de risco para doenças cardiovasculares. Através da curva receiver operating characteristic entre a aptidão cardiorrespiratória e as referências de fatores de risco para doenças cardiovasculares foram identificados os valores dos pontos de corte. Adicionalmente foi utilizada a análise bivariada seguida do cálculo de razão de chances (odds ratio-OR) para identificar quanto os indivíduos que não atenderam os pontos de corte propostos tinham a mais de chance de apresentar fatores de risco para doenças cardiovasculares. Os resultados indicaram que, dentre as propostas analisadas, a que foi sugerida neste estudo apresentou melhores ajustamentos entre sensibilidade e especificidade. Os resultados da análise bivariada mostraram que indivíduos que não atenderam os pontos de corte propostos têm maiores chances de apresentarem fatores de risco para doenças cardiovasculares.

Palavras-chave: aptidão cardiorrespiratória, teste de campo, pontos de corte, escolares, curva ROC.

\begin{abstract}
The main objective of the study was to analyze and propose news cardiorespiratory fitness cut offs points for 7 to 12-year old school students. The sample was composed of 1,413 students. Cardiorespiratory fitness was measured by the 9-minutes walking/running test. Total cholesterol and systolic and diastolic blood pressure were measured and with their results cardiovascular risk factors reference were designed. The Receiver Operating Characteristic curve between cardiorespiratory fitness and cardiovascular risk factors reference identified the cut offs point values. The bivariate analysis and odds ratio (OR) calculation was used to identify to what extent the individuals that did not reach the proposed cut offs points could show cardiovascular risk factors. The results showed that the cut offs points suggested in this study presented the best balance between sensibility and specificity. The bivariate analysis results showed increased chance of cardiovascular risk for the individuals that did not reach the cut offs points compared to those individuals who did.
\end{abstract}

Keywords: cardiorespiratory fitness, field test, cut offs points, school students, roc curve.

\section{INTRODUÇÃO}

A aptidão cardiorrespiratória $(A p C)$ é reconhecida como fator de proteção contra doenças cardiovasculares (DCV) em adultos ${ }^{(1,2)}$. Evidências de estudos científicos têm sugerido que tais doenças iniciam na infância e adolescência(3). Diante disto, parece fundamental a avaliação e acompanhamento da ApC nesta faixa etária.

A principal forma de medida da ApC é o consumo máximo de oxigênio por ergoespirometria. Contudo, tal procedimento não se torna viável quando o objetivo é a avaliação populacional, já que necessita de material específico, pessoal especializado, e demanda tempo relativamente grande para cada avaliação. Desta forma, a alternativa mais adequada é a utilização de testes de campo.

Os testes de campo para ApC mais utilizados em estudos que envolvem crianças e adolescentes são o teste de corrida/caminhada de nove minutos ${ }^{(4)}$, o teste de corrida/caminhada de uma milha ${ }^{(4)}$ e o teste vai-e-vem de 20 metros (20-m shuttle run test) ${ }^{(5)}$. No Brasil, o teste que tem sido mais utilizado é o de corrida/caminhada de nove minutos, sendo a forma de medida da ApC em vários estudos ${ }^{(6-9)}$. 
No que diz respeito à avaliação dos níveis de $A p C$ de crianças e adolescentes, duas alternativas de critérios de referência de instituições internacionais são frequentemente utilizadas. O Fitnessgram ${ }^{(10)} \mathrm{e} \mathrm{o}$ Physical Best ${ }^{(11)}$. Com relação à avaliação da ApC de crianças e adolescentes a partir de propostas nacionais, duas alternativas de pontos de corte aparecem como possibilidades ${ }^{(7-12)}$, porém, ainda sem muita expressão em sua utilização se comparadas às duas alternativas internacionais.

Os pontos de corte para a ApC de crianças e adolescentes propostos pela literatura merecem algumas considerações. Do ponto de vista metodológico, as propostas do Fitnessgram ${ }^{(10)}$ e o Physical Best ${ }^{(11)}$ foram baseadas nos valores que em adultos apresentam menor probabilidade de desenvolvimento de DCV ${ }^{(10,11)}$, mesmo com justificativas teóricas consistentes, devem ser utilizadas com cautela por não apresentar validação empírica. Também com necessidade de cautela em sua utilização, por não apresentar validação empírica, a proposta sugerida pelo Proesp-BR ${ }^{(7)}$ foi feita presumindo-se que indivíduos com índices inferiores de ApC apresentam maiores probabilidades de possuir fatores de riscos para as DCV, propondo o percentil 20, por sexo e idade, como ponto de corte.

Tal validação empírica poderia ser realizada verificando se os pontos de corte sugeridos pelas três instituições apresentam associação com fatores de risco para DCV. Neste sentido, utilizando 281 escolares de 15 a 18 anos de idade de Londrina/PR, Guedes et al. ${ }^{(12)}$ avaliaram a validade dos pontos de corte propostos pelo Physical Best para a ApC a partir da associação com fatores de risco para DCV. Além disto, a partir da análise de curva ROC, os autores propuseram pontos de corte para esta variável a partir dos valores do teste em sua amostra que apresentaram o melhor equilíbrio entre sensibilidade e especificidade.

Mesmo utilizando uma referência elaborada a partir de fatores de risco para DCV para associação com a ApC, e desta forma uma validação empírica, o estudo de Guedes et al. ${ }^{(12)}$, também deve ser analisado com cautela. $O$ estudo de Guedes et al. ${ }^{(12)}$, foi feito com o agrupamento de adolescentes de 15 a 18 anos, sugerindo um mesmo ponto de corte para as três idades, restringindo o uso apenas para a avaliação da ApC de indivíduos desta faixa etária, não havendo a possibilidade de avaliação de idades mais precoces, nas quais, de acordo com a literatura(3), inicia o desenvolvimento dos fatores de risco para DCV.

Considerando as informações acima apresentadas e discutidas, parece importante que sejam testadas a capacidade de triagem de crianças e adolescentes com fatores de risco para DCV pelos pontos de corte para ApC propostos pela literatura. Além disto, parece importante o desenvolvimento de propostas de pontos de corte para ApC que utilizem como referência fatores de risco para DCV, e desta forma apresentem validação empírica. Diante disto, os objetivos do presente estudo são: a) propor pontos de corte para ApC de escolares; b) avaliar o desempenho de pontos de corte para a ApC propostos pela literatura como instrumento de triagem na identificação de escolares com fatores de risco para DCV.

\section{MATERIAIS E MÉTODOS}

Este estudo transversal de base escolar foi realizado com escolares de sete a 12 anos de idade dos dois sexos, matriculados nas redes de ensino municipal, estadual e privada da cidade de Caxias do Sul/RS. A população de escolares de sete a 12 anos de idade no ano de 2005, de acordo com dados da Secretaria Estadual de Educação, foi de 33.241 escolares. Com base em estudos análogos ${ }^{(13,14)}$, foi estimada para o cálculo de tamanho da amostra uma prevalência média de hipertensão arterial e hipercolesterolemia de $20 \%$. Com um intervalo de confiança de $95 \%$, um poder de $80 \%$ e um erro de estimativa de $3 \%$, seriam necessárias 669 crianças. Utilizando-se um efeito de delineamento 2 para controle de fatores de confusão, acrescido de mais 15\% para suprir possíveis perdas e recusas, foi estimada a necessidade de avaliar 1.573 crianças. Das 1.573 crianças estimadas para o estudo, 1.413 realizaram as medidas de pressão arterial; 1.370 realizaram o teste de ApC; 1.294 permitiram a realização da coleta de sangue. O critério de amostragem adotado foi probabilístico por conglomerados, em que cada escola foi considerada um conglomerado. Todas as escolas do município (153) participaram do sorteio tendo as mesmas chances de participarem do estudo de acordo com o número de alunos matriculados na faixa etária de sete a 12 anos. Todos os escolares que participaram da composição da amostra apresentaram o Termo de Consentimento Livre e Esclarecido. O estudo foi aprovado pelo comitê de ética da Universidade Luterana do Brasil (protocolo 2006-365H). A coleta de dados ocorreu no período de abril a agosto de 2007.

A ApC dos escolares foi medida através do teste de corrida/caminhada de nove minutos seguindo as orientações do Proesp-BR ${ }^{(14)}$. $O$ teste consiste em percorrer a maior distância possível em um período de tempo de nove minutos. Para a análise por critérios de referência da ApC, foram utilizados os pontos de corte propostos pelo Fitnessgram ${ }^{(10)}$, pelo Physical Best ${ }^{(11)}$, e pelo Proesp-BR ${ }^{(14)}$. É importante destacar que os pontos de corte propostos pelo Fitnessgram e pelo Physical Best foram criados originalmente para a avaliação do teste de corrida/caminhada de uma milha. Assim, os pontos de corte propostos por estas duas instituições foram adaptadas para o teste de nove minutos, seguindo a atitude utilizada por outros estudos ${ }^{(6,8)}$.

Os fatores de risco para DCV medidos nos escolares foram o colesterol total (CT), a pressão arterial sistólica (PAS) e a pressão arterial diastólica (PAD). Os valores de colesterol total (CT) foram obtidos mediante utilização do monitor portátil Accutrend ${ }^{\circledR}$ GCT (Roche Diagnostics). 0 método de coleta seguiu as instruções preconizadas pelo fabricante. Os intervalos de medição são de 150-300mg/dl e o tempo necessário para a medição é de 180 segundos. O sangue foi retirado através da punção da face palmar da falange distal do dedo anelar através do lancetador (Softclix Pró) apropriado para usuários diferentes e respectivas lancetas descartáveis, graduado de um a três em grau crescente de profundidade de penetração (profundidade: $1=$ menor; $2=$ média; 3 = maior). Como padronização foi utilizado o grau dois de penetração para todos os exames. Para início do procedimento, o dedo deveria estar limpo e, após a punção, o avaliado receberia um algodão para pressionar sobre o local. Não foi exigido que os escolares estivessem em jejum, já que o CT não apresenta variação significativa com o indivíduo estando ou não em jejum ${ }^{(15)}$

A utilização desta forma de medida do CT (monitor portátil Accutrend ${ }^{\circledR}$ GCT Roche Diagnostics) e deste procedimento (não jejum) foi utilizada em campanha no Brasil, pela Sociedade Brasileira de Cardiologia em mais de 81 mil indivíduos ${ }^{(16)}$. Foi considerado colesterol total "desejável" valores menores que 170mg/dl, colesterol total "limítrofe" valores entre 170 e 199mg/dl e colesterol total "aumentado" valores iguais ou maiores que $200 \mathrm{mg} / \mathrm{dl}$, de acordo com as III Diretrizes Brasileiras Sobre Dislipidemias ${ }^{(15)}$.

As medidas da pressão arterial (PA) foram realizadas em uma sala onde três avaliadores previamente treinados e capacitados para a coleta se dispunham separadamente uns dos outros. Os estetoscópios (pediátricos) e os manguitos (pediátricos) eram da marca Becton Dickinson $^{\circledR}$ e os esfignomanômetros das marcas Cardiomed ${ }^{\circledR}$ e Oxigen ${ }^{\circledR}$, todos aferidos pelo Instituto Nacional de Metrologia. As crianças não haviam feito atividade física nos 30 minutos precursores, nem estavam de bexiga cheia. As medidas foram tomadas no braço direito com a criança sentada e bem relaxada. A PAS foi determinada no aparecimento do primeiro som de Korotkof e a PAD no desaparecimento do 
mesmo. Foram realizadas três medidas intervaladas por três minutos. Para a categorização da pressão arterial, levou-se em consideração o sexo, a idade e o percentil da estatura. Foram consideradas normais as PAS e PAD menores que os valores correspondentes ao percentil 90, e crianças com níveis pressóricos sistólico e/ou diastólico maiores ou iguais ao percentil 90 e menor que 95 foram consideradas préhipertensos. As crianças que atingiram valores acima do percentil 95 foram consideradas supostamente hipertensas, conforme a tabela do $4^{\circ}$ Relatório do National High Blood Pressure Education Program ${ }^{(17)}$.

Com relação ao tratamento dos dados, para a proposição dos pontos de corte para a ApC, foi utilizada a curva Receiver Operating Characteristic (ROC). Este procedimento necessita de uma variável "referência" (variável dicotômica) e uma variável "teste" (no caso do presente estudo a ApC). Para tanto foram criadas nove possibilidades de referência a partir dos fatores de risco para DCV dicotomizados da seguinte maneira: 1) CT "desejável/limítrofe" e "aumentado"; 2) CT "desejável" e "limítrofe/aumentado"; 3) PAS "normal/pré-hipertenso" e "hipertenso"; 4) PAS "normal" e "pré-hipertenso/hipertenso"; 5) PAD "normal/pré-hipertenso" e "hipertenso"; 6) PAD "normal" "pré-hipertenso/hipertenso"; 7) escore de fatores de risco para DCV $=5$ (escore $D C V \geq 5$ ); 8) escore $\mathrm{DCV} \geq 6, \mathrm{e} ;$ 9) escore $\mathrm{DCV} \geq 7$.

Os três escores de fatores de risco para DCV foram criados a partir de um somatório que levou em consideração a classificação do CT pelas III DBSD ${ }^{(15)}$, e da PAS e PAD pela NHBPEP(17), em que a inexistência do fator de risco (CT = desejável; PAS e PAD = normal) recebeu o valor "1", a existência da classificação intermediária do fator de risco (CT = limítrofe; PAS e PAD = pré-hipertenso) recebeu o valor "2", e a existência do fator de risco (CT = aumentado; PAS e PAD = hipertenso) recebeu o valor "3". Após a criação das nove referências de fatores de risco para DCV foi determinada a curva ROC entre a ApC, por sexo e idade, e cada uma das nove referências. A referência que na média entre as idades, por sexo, apresentou a maior área sob a curva ROC foi escolhida como a referência de fatores de risco para DCV. As referências que apresentaram a maior área sob a curva ROC foram o escore $D C V \geq 6(0,504)$ e o escore $D C V \geq 7(0,558)$ para meninos e meninas, respectivamente.

Com as referências para os fatores de risco para DCV definidas foram identificados os valores específicos de ApC em cada idade e nos dois sexos que apresentaram o melhor ajustamento entre sensibilidade e especificidade, identificando assim os pontos de corte para ApC a partir dos valores apresentados por nossa amostra. A análise quanto à validade dos pontos de corte para ApC, sugeridos pela literatura e pela amostra do presente estudo na tentativa de identificar indivíduos com chances aumentadas de apresentar fatores de risco para as DCV, foi realizada a partir da análise bivariada entre a ApC dos escolares categorizados por cada uma das propostas de pontos de corte e as referências que apresentaram a maior área sob a curva ROC seguidos dos cálculos de sensibilidade e especificidade. Com o intuito de identificar quanto os indivíduos que não atenderam ao ponto de corte para ApC em cada uma das propostas estudadas apresentam a mais de chance de portarem fatores de risco para as DCV que seus pares que atenderam o valor do ponto de corte, foi utilizada análise bivariada seguida do cálculo de razão de chances (odds ratio-OR). As análises para a identificação dos pontos de corte foram realizadas levando-se em consideração uma área sob a curva ROC mínima de $50 \%(0,5)^{(18)}$ e as análises bivariadas um intervalo de confiança de 95\% (IC 95\%) e P $\leq$ 0,05. Todas as análises foram realizadas no programa SPSS for Windows versão 13.0.

\section{RESULTADOS}

Os resultados da análise da curva $\mathrm{ROC}$ entre a $\mathrm{ApC}$ e as referências de fatores de risco para DCV, por sexo e idade, estão apresentadas na tabela 1. Analisando os resultados, percebemos que aos oito, nove, 10, e 12 anos nos meninos e aos oito, nove e 12 anos nas meninas, o valor da área sob a curva ROC não atingiu 50\%. Contudo, os valores nestas idades, com exceção dos nove anos nas meninas, aproximaramse consideravelmente do valor de 50\%. Além disso, a média entre as idades da área da curva ROC é de 0,504 (50,4\%) para meninos, e de 0,558 $(55,8 \%)$ para meninas, ultrapassando o valor mínimo estabelecido de 50\%. Os valores de sensibilidade demonstraram moderada capacidade de identificar escolares classificados como portadores de fatores de risco para DCV que não atenderam aos pontos de corte (verdadeiros positivos). Os valores de especificidade também apresentaram moderada capacidade de identificar escolares classificados como não portadores de fatores de risco para DCV que atenderam os pontos de corte (verdadeiros negativos).

Na tabela 2 são apresentados os valores de sensibilidade e especificidade calculados a partir da associação entre os pontos de corte para a ApC propostos pelo Fitnessgram ${ }^{(10)}$, pelo Physical Best ${ }^{(11)}$, pelo Proesp-BR ${ }^{(7)}$, e pelo presente estudo com as referências de fatores de risco para DCV. Analisando os resultados, nota-se que a proposta que apresenta o melhor equilíbrio entre os valores de sensibilidade e especificidade é a do presente estudo, configurando-se como a proposta mais indicada de pontos de corte para a ApC.

Quando a ApC é categorizada pelos pontos de corte propostos pelo presente estudo, associada às referências de fatores de risco para DCV através da análise bivariada seguida do cálculo da razão de chances, nota-se que para os dois sexos os escolares que não atingiram as distâncias indicadas, como pontos de corte apresentam maior chance de portarem fatores de risco para DCV (tabela 3). Nos meninos, aqueles que não atenderam o ponto de corte, a chance de apresentarem fatores de risco para DCV é 98,1\% maior que aqueles que atenderam. Nas meninas, a chance é $64,9 \%$ maior de apresentar fatores de risco para DCV naquelas que não atenderam o ponto de corte.

Tabela 1. Resultados da curva ROC entre a ApC e as referências de fatores de risco para DCV.

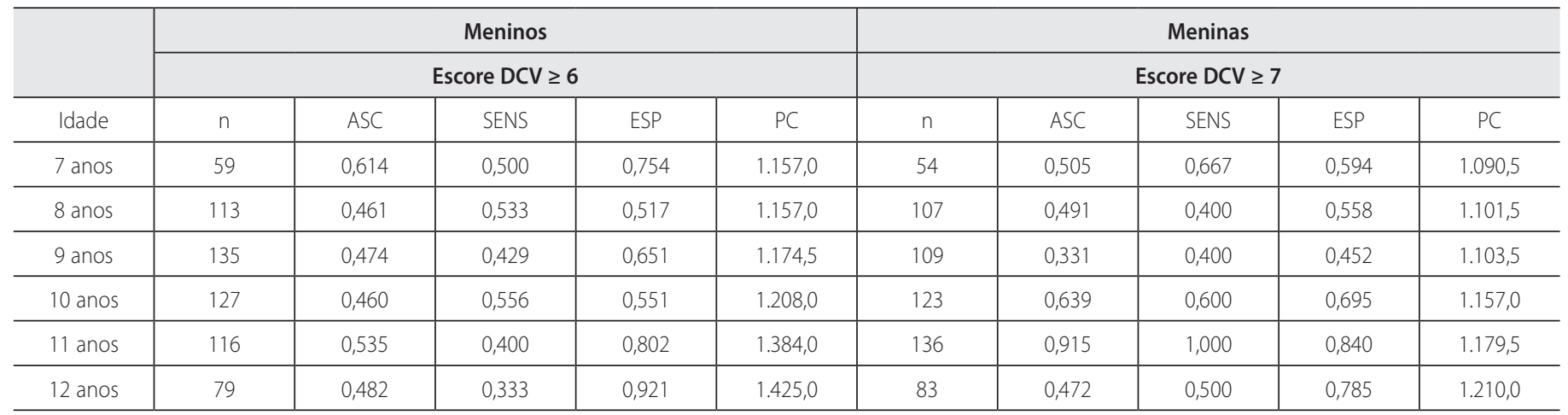

$\mathrm{N}=$ amostra; $\mathrm{ASC}$ = área sob a curva ROC; SENS = sensibilidade; ESP = especificidade; $\mathrm{PC}=$ ponto de corte referente à distância em metros a ser percorrida para o avaliado ter chance reduzida de apresentar fatores de risco para DCV. 


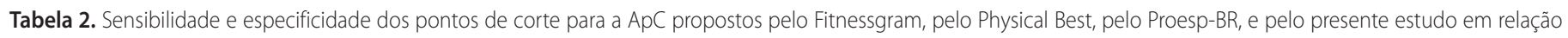
ao escore de $D C V \geq 6$ (meninos) e ao escore de $D C V \geq 7$ (meninas).

\begin{tabular}{|c|c|c|c|c|}
\hline \multirow{2}{*}{$\frac{\text { PC }}{\text { sugeridos }}$} & \multicolumn{2}{|c|}{ Meninos } & \multicolumn{2}{|c|}{ Meninas } \\
\hline & SENS (IC95\%) & ESP (IC95\%) & SENS (IC95\%) & ESP (IC95\%) \\
\hline Physical Best & $0,926(0,84-1,00)$ & $0,065(0,04-0,08)$ & $0,793(0,64-0,94)$ & $0,113(0,09-0,14)$ \\
\hline Fitnessgram & $0,731(0,59-0,87)$ & $0,338(0,30-0,38)$ & $0,379(0,20-0,56)$ & $0,509(0,47-0,55)$ \\
\hline Proesp-BR & $0,341(0,19-0,49)$ & $0,692(0,65-0,73)$ & $0,275(0,11-0,44)$ & $0,763(0,73-0,80$ \\
\hline Presente estudo & $0,512(0,35-0,67)$ & $0,566(0,52-0,61)$ & $0,586(0,40-0,77)$ & $0,622(0,54-0,63)$ \\
\hline
\end{tabular}

Tabela 3. Resultados da análise bivariada e do cálculo de razão de chances entre as referências de fatores de risco para DCV e a ApC categorizada pelos pontos de corte propostos por nosso estudo.

\begin{tabular}{c|c|c|c|c}
\hline sexo & $\mathbf{n}(\%)$ & OR & IC (95\%) & P \\
\hline Meninas (escore DCV $\geq 7)$ & & & & \\
\hline Atenderam ao PC & $375(61,3)$ & 1,00 & - & - \\
\hline Não atenderam ao PC & $237(38,7)$ & 1,649 & $1,40-1,942$ & 0,000 \\
\hline Meninos (escore DCV $\geq 6)$ & & & & \\
\hline Atenderam ao PC & $353(56,1)$ & 1,00 & - & - \\
\hline Não atenderam ao PC & $276(43,9)$ & 1,981 & $1,67-2,33$ & 0,000 \\
\hline
\end{tabular}

n (\%): número amostral absoluto e (percentual); OR: odds ratio; IC (95\%): intervalo de confiança de 95\%; P: significância.

\section{DISCUSSÃO}

Considerando as evidências de que níveis adequados de ApC servem como fator de proteção contra DCV e que tais doenças iniciam na infância e adolescência, identificar valores que abaixo deles aumente a chance de escolares desenvolverem fatores de risco para DCV parece fundamental, constituindo-se como instrumento para prevenção primária. Além disso, é importante identificar estes valores em testes de campo, uma vez que para medidas populacionais da ApC métodos diretos são inviáveis. No Brasil, o teste de campo para a ApC que mais se destaca é o de corrida/caminhada de nove minutos. Tal teste é recomendado pelo Proesp-BR ${ }^{(7)}$, projeto de abrangência nacional com apoio do Ministério do Esporte, por ser um teste de fácil aplicação e por ter o tempo de duração definido, permitindo ao avaliador saber quando o teste será encerrado. Desta forma, o presente estudo configura-se como a primeira tentativa nacional de proposição de pontos de corte para ApC, medida por teste de campo, em uma amostra populacional de base escolar que apresente validação empírica através da associação com fatores de risco para DCV.

Os valores médios da área sob a curva ROC encontrado no presente estudo são similares aos encontrados no estudo de Guedes et al.(12), mas um pouco inferiores aos encontrados no estudo de Ruiz et al. ${ }^{(19)}$. Não obstante, é importante ressaltar que no estudo de Guedes et al.(12) a medida de ApC foi feita, assim como no presente estudo, através de um teste de campo (corrida/caminhada da milha), enquanto que no estudo de Ruiz et al. ${ }^{(19)}$ foi utilizada a medida direta de consumo de oxigênio. Tais características talvez possam ajudar a explicar a similaridade entre as áreas sob a curva ROC o estudo de Guedes et al. ${ }^{(12)}$ e a superioridade do estudo de Ruiz et al. ${ }^{(19)}$.

Os valores de sensibilidade e especificidade apresentados pelas diferentes propostas de pontos de corte para ApC não podem ser considerados altos. Contudo, considerando os inúmeros fatores que contribuem para a variação dos resultados das variáveis que foram utilizados em nosso estudo para a elaboração das referências de fatores de risco para DCV, como nível de atividade física habitual, estado nutricional, hábitos alimentares, histórico familiar, nível socioeconômico e estágio maturacional|(13,20-23) é compreensível que não tenha sido encontrado valores mais altos e equilibrados de sensibilidade e especificidade. A não inserção de tais variáveis pode ser considerada como uma limitação do presente estudo. Por outro lado, se valores elevados de sensibilidade e especificidade não foram encontrados, os pontos de corte propostos no presente estudo apresentam considerável equilíbrio entre sensibilidade e especificidade, podendo ser considerados como propostas de pontos de corte para ApC possíveis para a triagem de escolares com maior chance de apresentarem fatores de risco para DCV.

Além de apresentar melhor equilíbrio entre sensibilidade e especificidade, os pontos de corte para ApC de crianças e adolescentes, sugeridos por nosso estudo, apresentam outras características que os distingue daqueles propostos pela literatura. A validação empírica através da associação da ApC com fatores de risco para DCV. As propostas do Fitnessgram, do Physical Best e do Proesp-BR utilizaram outras formas para sugerir seus pontos de corte para a ApC.

As propostas do Fitnessgram e do Physical Best foram desenvolvidas levando em consideração os valores de consumo máximo de oxigênio que em adultos estão mais associados com problemas cardiovasculares e todos os seus fatores de risco e extrapolados para um teste de campo (teste de corrida/caminhada da milha) para crianças e adolescentes levando em consideração o desenvolvimento do consumo de oxigênio e de economia de corrida durante a infância e adolescência. Com isto, os pesquisadores conseguiram identificar a velocidade na qual crianças e adolescentes deveriam realizar a atividade para que tivessem um consumo de oxigênio considerado adequado para a saúde. Com a velocidade definida e a distância conhecida (uma milha), foi necessário apenas calcular o tempo para propor os valores de ponto de corte ${ }^{(24)}$. Como as propostas do Fitnessgram e do Physical Best se baseiam na velocidade de deslocamento durante o teste, seus pontos de corte para a ApC permitem que sejam adaptados para testes de tempo fixo (teste de corrida/caminhada de nove minutos).

A proposta do Proesp-BR, única proposta nacional de pontos de corte para a ApC de crianças e adolescentes em diferentes idades, foi criada levando em consideração a distribuição de valores dos resultados do teste de corrida/caminhada de nove minutos em uma amostra representativa de todo o país. O percentil 20 foi estabelecido como ponto de corte levando em consideração o sexo e as idades. Tal escolha foi feita por presumir-se que indivíduos com desempenhos mais baixos no teste apresentam maiores probabilidades de portarem fatores de riscos à doenças relacionadas ao sedentarismo nesta categoria(7).

A tabela 4 apresenta os valores de pontos de corte para ApC propostos pelo Fitnessgram, pelo Physical Best, pelo Proesp-BR e por nosso estudo. É possível observar que, dentre as quatro propostas, a que apresenta os valores mais altos de pontos de corte é a do Physical Best, e a que apresenta valores mais baixos é a do Proesp-BR. Tais características ajudam a compreender os elevados valores de sensibilidade apresentados pela proposta do Physical Best e de especificidade do Proesp-BR, mas com baixos valores de especificidade e sensibilidade, respectivamente. Além disso, devido à maior proximidade entre os valores propostos pelo Fitnessgram e pelo presente estudo, é possível entender porque o equilíbrio entre os valores de sensibilidade e especificidade apresentados pela proposta do Fitnessgram foi o que mais se aproximou do equilíbrio 
Tabela 4. Valores de pontos de corte (metros) para o teste de corrida/caminhada de nove minutos das diferentes propostas estudadas.

\begin{tabular}{c|c|c|c|c|c|c|c|c}
\hline Idade & \multicolumn{2}{|c|}{ Physical Best } & \multicolumn{2}{c|}{ Fitnessgram } & \multicolumn{2}{c}{ Proesp-BR } & \multicolumn{2}{c}{ Presente estudo } \\
\hline (anos) & masculino & feminino & masculino & feminino & masculino & feminino & masculino & feminino \\
\hline 7 & $1.316,4$ & $1.206,7$ & $1.034,3$ & 965,4 & 930,0 & 886,0 & $1.157,0$ & $1.090,5$ \\
\hline 8 & $1.448,1$ & $1.259,2$ & $1.113,9$ & 998,68 & 986,0 & 922,0 & $1.157,0$ & $1.101,5$ \\
\hline 9 & $1.448,1$ & $1.316,4$ & $1.206,7$ & $1.113,9$ & $1.040,0$ & 953,0 & $1.174,5$ & $1.103,5$ \\
\hline 10 & $1.524,3$ & $1.316,4$ & $1.316,4$ & $1.206,7$ & $1.093,0$ & 979,0 & $1.208,0$ & $1.157,0$ \\
\hline 11 & $1.609,0$ & $1.316,4$ & $1.316,4$ & $1.206,7$ & $1.144,0$ & $1.000,0$ & $1.384,0$ & $1.179,5$ \\
\hline 12 & $1.609,0$ & $1.316,4$ & $1.448,1$ & $1.259,2$ & $1.194,0$ & $1.017,0$ & $1.425,0$ & $1.210,0$ \\
\hline
\end{tabular}

entre os valores de sensibilidade e especificidade apresentados pela proposta do presente estudo (tabela 2).

A diferença existente entre os valores de pontos de corte das propostas do Physical Best e do Fitnessgram existem, mesmo ambas tendo sido elaboradas seguindo os mesmos procedimentos, pois os valores considerados adequados para saúde de consumo de oxigênio em crianças e adolescentes não foram os mesmos. As duas propostas adotaram como valores de consumo máximo de oxigênio mínimo, considerado adequado para saúde, os propostos por Cooper ${ }^{(25)}$ para adultos, $42 \mathrm{~mL} \cdot \mathrm{Kg}^{-1} \cdot \mathrm{min}^{-1}$ e $35 \mathrm{~mL} \cdot \mathrm{Kg}^{-1} \cdot \mathrm{min}^{-1}$ para homens e mulheres, respectivamente. O Fitnessgram manteve estes valores para as crianças e adolescentes, respeitando o desenvolvimento da economia de corrida que ocorre neste período. O Physical Best, por outro lado, aumentou em $8 \mathrm{~mL} \cdot \mathrm{Kg}^{-1} \cdot \mathrm{min}^{-1} \mathrm{Os}$ valores baseado em algumas evidências que sugeriam que o consumo de oxigênio tende a diminuir da infância e adolescência para a vida adulta. Assim, os valores dos pontos de corte propostos pelo Physical Best acabaram ficando mais exigentes ${ }^{(24)}$.

A possibilidade de utilização dos pontos de corte para ApC propostos em nosso estudo, indicada por apresentar o melhor equilíbrio entre sensibilidade e especificidade entre os pontos de corte estudados, fica reforçada pelos resultados disponibilizados pela análise bivariada. Esta

\section{REFERÊNCIAS}

1. Lee CD, Blair SN, Jackson AS. Cardiorespiratory fitnes, body composition, and all-cause and cardiovascular disease mortality in men. Am J Clin Nutr 1999;96:373-80.

2. Lamonte MJ, Barlow CE, Jurca R, Kampert JB, Church TS, Blair SN. Cardiorespiratory Fitness Is Inversely Associated With the Incidence of Metabolic Syndrome: A Prospective Study of Men and Women Circulation 2005;12:505-12

3. Raitakari OT, Juonala M, Kähönen M, Taittonen L, Tomi Laitinen T, Mäki-Torkko N, et al. Cardiovascular Risk Factors in Childhood and Carotid Artery Intima-Media Thickness in Adulthood: The Cardiovascular Risk in Young Finns Study. JAMA 2003;290:2277-83.

4. AAHPERD. Health-related physical fitness test manual. Reston, Virginia: American Alliance For Health, Physical Education and Recreation and Dance, 1980.

5. Léger LA, Lambert J. A maximal multistage 20-m shuttle run test to predict $\mathrm{VO}_{2}$ max. Eur J Appl Physiol 1982;49:1-12.

6. Bergmann GG, Araújo MLB, Lorenzi T, Garlipp D, Gaya A. Alteração Anual no Crescimento e na Aptidão Física Relacionada à Saúde de Escolares. Rev Bras Cineantropom Desempenho Hum 2005;7:55-61.

7. Gaya A, Silva GMG. PROESP-BR: Observatório Permanente dos Indicadores de saúde e fatores de prestação esportiva em crianças e jovens. Manual de Aplicação de Medidas e Testes, Normas e Critérios de Avaliação, 2007 (disponível em http://www.proesp.ufrgs.br) acessado em 20 de outubro de 2007.

8. Bergmann MLB, Bergmann GG, Halpern R. Perfil Lipídico, de Aptidão Cardiorrespiratória, e de Composição Corporal de uma Amostra de Escolares de 8a Série de Canoas/RS. Rev Bras Med Esporte 2008;14:22-7.

9. Dumith SC, Azevedo Júnior MR, Rombaldi AJ. Aptidão Física Relacionada à Saúde de Alunos do Ensino Fundamental do Município de Rio Grande, RS, Brasil. Rev Bras Med Esporte 2008;14:454-9.

10. 10.Institute for Aerobic Research. Fitnessgram User's Manual. Dallas, Texas, Institute for aerobics Research, 1987.

11. AAHPERD. Physical Best. Reston, Virginia: American Alliance for Health, Physical Education and Recreation and Dance, 1988.

12. Guedes DP, Guedes JERP, Barbosa DS, Oliveira JA. Aptidão física relacionada à saúde e fatores de risco predisponentes às doenças cardiovasculares em adolescentes. Rev Port Cien Desp 2002;2:31-46.

13. Coronelli CLS, Moura EC. Hipercolesterolemia em escolares e seus fatores de risco. Rev Saúde Pública 2003;37:24-31.

14. Moura AA, Silva MAM, Ferraz MRMT, Rivera IR. Prevalência de pressão arterial elevada em escolares e adolescentes de Maceió. J Pediatr 2004;80:35-40.

15. IIIDiretrizes brasileiras sobre dislipidemias e diretriz de prevenção da aterosclerose do Departamento de aterosclerose da Sociedade Brasileira de Cardiologia. Arq Bras Cardiol 2001;77:1-48.

16. Martinez TLR, Santos RD, Armaganijan D, Torres KP, Loures-Vale A, Magalhães ME, Lima JC, Moriguchi E, Amodeo C, Ortiz J. Campanha Nacional de Alerta Sobre o Colesterol Elevado. Determinação do Nível análise identificou que escolares que não alcançaram os pontos de corte propostos apresentaram maiores chances $(O R=1,981 ; \mathrm{IC}=1,67-2,33$ meninos; $O R=1,649 ; \mid C=1,40-1,942$ - meninas) de portarem fatores de risco para DCV que aqueles que alcançaram. Os resultados da análise de regressão logística vão ao encontro de evidências disponíveis na literatura que indicam associação entre baixos valores de ApC e a presença de fatores de risco para DCV em crianças e adolescentes ${ }^{(19,26-30)}$.

\section{CONCLUSÃO}

Frente aos resultados encontrados ficam evidências de que indivíduos com baixa ApC, medida pelo teste de corrida/caminhada de nove minutos, têm maiores chances de apresentar fatores de risco para DCV em comparação àqueles com melhores resultados. Além disso, os pontos de corte propostos pelo presente estudo mostraram-se mais adequados que aqueles disponíveis na literatura para a identificação de escolares com chances aumentadas de portarem para fatores de risco para DCV. Desta forma, para a medida da ApC de escolares sugerimos a utilização do teste de corrida/caminhada de nove minutos e a avaliação pelos pontos de corte propostos no presente estudo.

Todos os autores declararam não haver qualquer potencial conflito de interesses referente a este artigo. de Colesterol de 81.262 Brasileiros Arq Bras Cardiol 2003:80:635-8.

17. NHBPEP. National High Blood Pressure Education Program Working Group on High Blood Pressure in Children and Adolescent. The Fourth report on the diagnosis, evaluation, end treatment of high blood pressure in children and adolescents. Pediatrics 2004;114:555-76.

18. Erdreich LS, Lee ET. Use of relative operating characteristics analysis in epidemiology: a method for dealing with subjective judgement. Am J Epidemiol 1981;114:649-62.

19. Ruiz JR, Ortega FB, Rizzo NC, Villa I, Hurtig-Wennlof A, Oja L, Sjostrom M. High Cardiovascular Fitness Is Associated with Low MetabolicRisk Score in Children: The European Youth Heart Study. Pediatr Res 2007;61:350-5

20. Forti N, Giannini SD, Diament J, Issa J, Fukushima J, Dal Bó C, Barreto ACP. Fatores de risco para doença Arterial coronariana em crianças e adolescentes filhos de coronariopatas jovens. Arq Bras Cardiol 1996;66:119-23.

21. Duarte JA, Ribeiro JC, Oliveira J, Mota J. The relationship between physical activity cholesterol levels in children and adolescents. Rev Bras Saude Mater Infant 2004;4:185-92.

22. Garcia FD, Terra AF, Queiroz AM, Correia CA, Ramos PS, Ferreira QT, et al. Avaliação de fatores de risco associados com elevação da pressăo arterial em crianças. J Pediatr 2004;80:29-34.

23. Ruiz JR, Ortega FB, Loit HM, Veidebaum T, Sjostrom M. Body fat is associated with blood pressure in school-aged girls with low cardiorespiratory fitness: The European Youth Heart Study. J Hypertens 2007;25:2027-34.

24. Cureton KJ, Warren GL. Criterion-Referenced Standards for Youth Health-Related Fitness Test: A Tutorial. Res Q Exerc Sport 1990;61:7-19.

25. Cooper KH. Aerobics. New York: Bantam Books, 1968.

26. Boreham C, Twisk J, Murray L, Savage M, Strain JJ, Cran G. Fitness, fatness, and coronary heart disease risk in adolescents: the Northern Ireland Young Hearts Project. Med Sci Sports Exerc 2001;33:270-4.

27. Brage S, Weddrkopp N, Ekelund U, Franks PW, Wareham NJ, Andersen LB, Froberg K. European Youth Heart Study (EYHS) Features of the metabolic syndrome are associated with objectively measured physical activity and fitness in Danish children: the European Youth Heart Study (EYHS). Diabetes Care 2004;27:2141-8.

28. Eisenmann JC, Welk GJ, Wickel EE, Blair SN. Combined influence of cardiorespiratory fitness and body mass index on cardiovascular disease risk factors among 8-18 year old youth: The Aerobics Center Longitudinal Study. Int J Pediatr Obes 2007;2:66-72.

29. Janssen I, Cramp WC. Cardiorespiratory Fitness Is Strongly Related to the Metabolic Syndrome in Adolescents. Diabetes Care 2007;30:2143-4.

30. Stabelini Neto A, Bozza R, Ulbrich AZ, Vasconcelos IQA, Boguszewski MCS, Campos W. Fatores de risco para aterosclerose associados à aptidão cardiorrespiratória e ao IMC em adolescentes. Arq Bras Endocrinol Metab 2008;52:1024-30. 